EPJ Web of Conferences 59, 05013 (2013)

DOI: $10.1051 /$ epjconf/20135905013

(C) Owned by the authors, published by EDP Sciences, 2013

\title{
Intensity dependence of electron gas kinetics in a laser corona
}

\author{
Martin Mašek ${ }^{\mathrm{a}}$ and Karel Rohlena \\ Institute of Physics, Academy of Sciences of the Czech Republic, Na Slovance 2, \\ 18221 Prague 8, Czech Republic
}

\begin{abstract}
In various experimental situations relevant to the laser fusion, such as plasma near the light entrance holes of hohlraum in the indirect drive experiments or more recently in the shock ignition direct drive a relatively long underdense plasma of corona type is encountered, which is subject to an intense nanosecond laser beam. The plasma is only weakly collisional and thus in the electron phase space a complicated kinetic evolution is going on, which is taking the electron gas fairly far from the thermal equilibrium and contributes to its unstable behaviour. These phenomena impede the absorption and thermalization of the incoming laser energy, create groups of fast electrons and also may lead to a nonlinear reflection of the heating laser beam. One of the key processes leading to the electron acceleration is the stimulated Raman scattering (SRS) in its non-linear phase. The SRS in the presence of electronion collisions requires a certain threshold intensity above which the mentioned non-dissipative phenomena can occur and develop to the stage, where they may become unpleasant for the fusion experiments. To assess this intensity limit a computational model has been developed based on the Vlasov-Maxwell kinetics describing such a plasma in $1 \mathrm{D}$ geometry. At a relatively high intensity of $10^{16} \mathrm{~W} / \mathrm{cm}^{2}$ a number of non-linear phenomena are predicted by the code such as a saturation of Landau damping, which is then translated in an unfavourable time dependence of the reflected light intensity and formation of accelerated electron groups due to the electron trapping. The purpose of the present contribution is to map the intensity dependence of this non-linear development with the aim of assessing its weight in fusion relevant situations.
\end{abstract}

\section{INTRODUCTION}

SRS can be characterized as a resonant decay of incident laser light into a scattered electromagnetic wave (EMW) and an electron plasma wave (EPW). In 1D we can distinguish two possibilities, forwardand back-scattering according to the propagation direction of the scattered EMW, when especially Raman backscattering (SRS-B) causes serious troubles in various experimental situations relevant to the laser fusion. The SRS-B growth rate $\gamma_{B}$ is known from the linear theory. Given the collisionless Landau damping rate $\gamma_{L}$ and electron-ion collision frequency $\nu_{e i}$ the instability threshold is $\gamma_{B}-\sqrt{\gamma_{L} \nu_{e i} / 2} \geq 0$. Combining it with the linear dispersion relation for the SRS-B we can localize the regions in the plasma corona with a strong growth of the instability. In this paper we concentrate on the fusion relevant situation, where the laser wavelength is $\lambda=0.4384 \mu \mathrm{m}$, the laser pulse duration is $\tau=400 \mathrm{ps}$, the focal spot diameter is $L=100 \mu \mathrm{m}$ and the electron temperature is $k_{B} T_{e}=4.44 \mathrm{keV}$. In such a plasma the strongest growth is observed in a thin region of outer corona and the instability threshold is to be expected expect above the laser intensity $P=10^{15} \mathrm{~W} / \mathrm{cm}^{2}$. The laser intensity dependence of $\gamma_{B}-\sqrt{\gamma_{L} \nu_{e i} / 2}$ is shown as the blue line in Fig. 1(b) with the fixed value of electron density $n_{e} / n_{c}=0.115$ and electron-ion collision frequency $v_{e i} / \omega_{p e}=0.01$. The plasma is then only slightly

\footnotetext{
ae-mail: masekm@fzu.cz
}

This is an Open Access article distributed under the terms of the Creative Commons Attribution License 2.0, which permits unrestricted use, distribution, and reproduction in any medium, provided the original work is properly cited. 

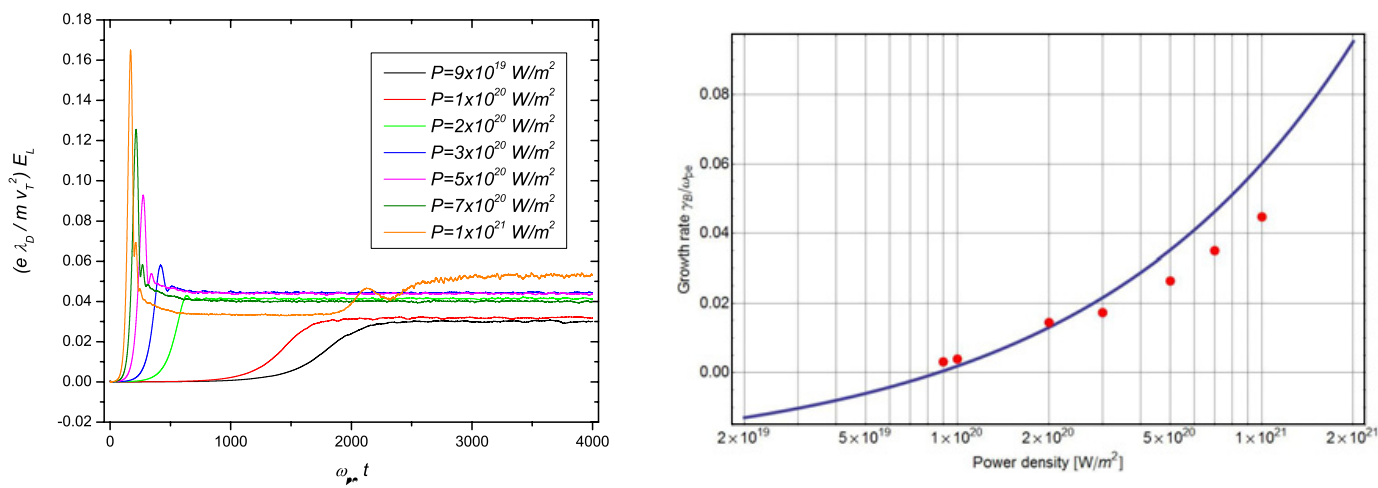

Figure 1. (a) Temporal evolution of resonant plasma wave mode from the full Vlasov-Maxwell simulation with different laser intensities. (b) SRS-B growth rate as a function of laser intensity (electron temperature is fixed at $k_{B} T_{e}=4.44 \mathrm{keV}$ ) obtained from the linear theory (blue line) is compared with the results of the Vlasov-Maxwell model (red points). (Note the SI units in the power density)

collisional, thus we can expect the main contribution to the instability damping from the wave-particle interaction, appearing in the linear regime as the Landau damping.

To extend the results of the linear theory, we developed a non-linear 1D kinetic model of electron gas, which numerically solves the Vlasov equation (1) simultaneously with the Maxwell equations (2-3):

$$
\begin{gathered}
\frac{\partial f}{\partial t}+v_{x} \frac{\partial f}{\partial x}+\frac{e}{m}\left(\frac{\partial \varphi}{\partial x}-\frac{e}{m} A_{y} \frac{\partial A_{y}}{\partial x}\right) \frac{\partial f}{\partial v_{x}}=v_{e i}\left(\frac{\partial\left(v_{x} f\right)}{\partial v_{x}}+<v_{x}^{2}>\frac{\partial^{2} f}{\partial v_{x}^{2}}\right), \\
{\left[\frac{\partial^{2}}{\partial x^{2}}-\frac{1}{c^{2}} \frac{\partial^{2}}{\partial t^{2}}-\frac{\omega_{p e}^{2}}{c^{2}} \frac{n_{e}}{n_{i}}\right] A_{y}=0} \\
\frac{\partial^{2} \varphi}{\partial x^{2}}=\frac{e}{m}\left(n_{e}-n_{i}\right) .
\end{gathered}
$$

The velocity in the perpendicular direction is replaced by the mean oscillatory velocity in the field of incident laser light $v_{y}=e A_{y} / m$. The vector potential has the only component $A_{y}$ in the perpendicular direction. We believe that this assumption is satisfactory for non-relativistic plasmas generated by a ns laser pulse, where the acceleration of electrons is done exclusively by the longitudinal field. The parametric instabilities are more likely present in long scale-length plasmas, which enables us to use a homogeneous periodic model to study kinetic effects connected with the Raman scattering. The natural and simple way how to solve the above set of equation (1-3) is to use the Fourier-Hermite transform method first described in [1]. It brings many advantages like a use of simplified FokkerPlanck collisional term on the right hand side of the Vlasov equation (1), which incorporates the collisions in a realistic manner with the entropy growth well defined physically $[2,3]$, in contrast to the mesh-based Vlasov simulations. The Fokker-Planck term provides a satisfactory stabilization of the method even with the use of realistic value of collisional frequency. Moreover, the reciprocal space renders the temporal evolution of resonant wave modes as an immediate result of the simulation. In the present paper we made use of the option of the method to set the Fourier spectrum as sparse as possible in order to study the kinetic effects connected with the pure Raman resonant mode only. Making the spectrum denser would introduce the side modes (connected e.g. with trapped particle instability) and thus complicate the physical situation. For a more detailed information see [4]. 

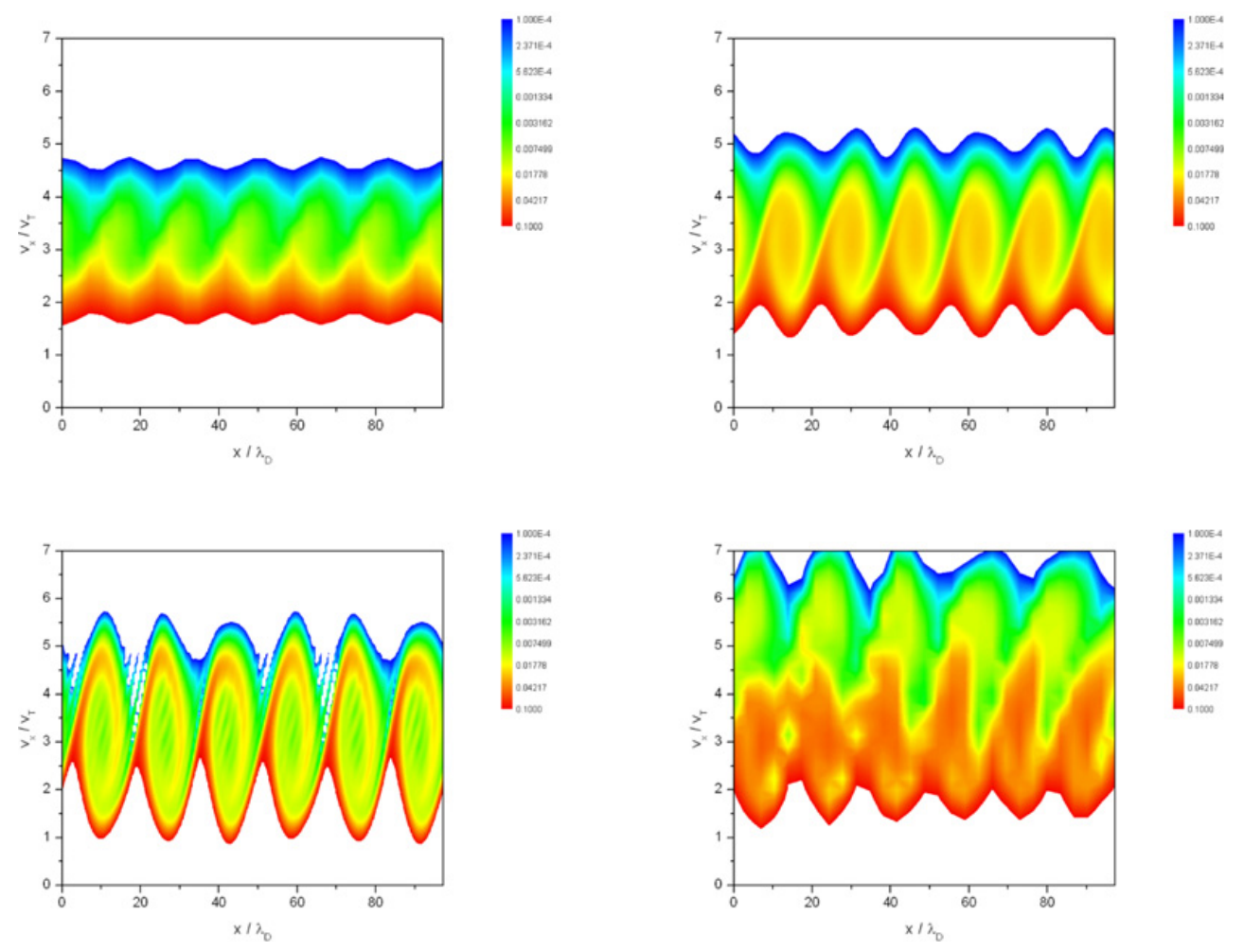

Figure 2. Snapshots of electron phase space (a) at $\omega_{p e} t=3200$ for $P=9 \times 10^{15} \mathrm{~W} / \mathrm{cm}^{2}$, (b) at $\omega_{p e} t=360$ for $P=5 \times 10^{16} \mathrm{~W} / \mathrm{cm}^{2}$, (c) at $\omega_{p e} t=160$ for $P=1 \times 10^{17} \mathrm{~W} / \mathrm{cm}^{2}$ and (d) at $\omega_{p e} t=2320$ for $P=1 \times$ $10^{17} \mathrm{~W} / \mathrm{cm}^{2} .\left(v_{T}=\sqrt{3 k_{B} T_{e} / m_{e}}\right)$.

\section{RESULTS OF NUMERICAL SIMULATION}

The laser intensity was varied in the interval from $P=1 \times 10^{15} \mathrm{~W} / \mathrm{cm}^{2}$ to $P=1 \times 10^{17} \mathrm{~W} / \mathrm{cm}^{2}$. Fig. 1(a) shows the temporal dependence of SRS-B EPW amplitude. In the fully non-linear case we found the collisional instability threshold to be slightly below $P=9 \times 10^{15} \mathrm{~W} / \mathrm{cm}^{2}$, when a weak growth with a fast saturation appeared. In the phase space picture it is seen that the electron plasma wave is not strong enough to trap a large amount of electrons (see Fig. 2(a)), so that the wave-particle interaction does not essentially participate in the temporal evolution of the wave modes. The saturation of the instability growth is caused by the wave-wave interaction of the participating waves (the ns pulse is pumping the energy into the plasma during the whole simulation time), the system is in a linear regime and the measured instability growth rate is well within the value as predicted by the linear theory (the first three red points lie near the blue curve in Fig. 1(b)). This situation lasts in our particular case till the laser intensity reaches a value around $P=2 \times 10^{16} \mathrm{~W} / \mathrm{cm}^{2}$. Above this a 'second threshold' connected with a fully non-linear wave-particle collisionless interaction appears. It is characterized by the fast amplitude growth (pumping laser is strong enough) followed by a fast collisionless damping of the resonant electrostatic wave mode as a consequence of strong electron trapping in the EPW troughs leading to a saturation. It is illustrated on the phase space plot in Fig. 2(b), where shortly after the EPW amplitude saturation at $\omega_{p e} t=360$ the fully developed structures of well trapped electrons are visible around the SRS-B EPW phase velocity for $P=5 \times 10^{16} \mathrm{~W} / \mathrm{cm}^{2}$.

The simulation was intentionally set to prevent a development of side modes connected with the trapped particle instability and thus an energy exchange between these modes was forbidden (compare 


\section{EPJ Web of Conferences}

[5]). We thus cannot observe a typical bursting behaviour of the instability caused by the interaction of these side modes with the trapped electrons. The only exception is the highest laser intensity visible in the temporal evolution of the SRS-B EPW starting at $\omega_{p e} t \approx 2000$ in Fig. 1(a). The half width of spectral broadening of the resonant mode in the case of trapped particle instability is $\delta k_{B} \approx \omega_{b f} / v_{p h B}$, where $v_{p h B}$ is the phase velocity of SRS-B EPW and $\omega_{b f}=\sqrt{e E_{B} k_{B} / m_{e}}$ is the bouncing frequency of trapped electrons. In this high intensity case the longitudinal electric field $E_{B}$ is high enough to allow $\delta k_{B}$ to bridge the gap between the neighbouring wave modes of the Fourier spectrum of numerical model. The onset of the trapped particle instability is evidenced by larger and deformed trapping regions in the phase space compared to the lower laser intensities (as seen in Fig. 2(d) compared to Fig. 2(b)). Correspondingly, the temporal evolution of EPW for $P=10^{17} \mathrm{~W} / \mathrm{cm}^{2}$ in Fig.1(a) is exceptional.

It is interesting to notice that the saturation level for the highest pumping laser intensity is lower in this regime. The obvious cause is the growing region of phase space affected by the propagating EPW and therefore a stronger wave-particle interaction leading to a lower resulting saturation level and also a lower Raman reflectivity.

The mentioned two regimes of the instability growth can also be easily recognized in Fig. 1(b), where the results of the numerical simulations are compared with the results of the linear theory $\gamma_{B}-\sqrt{\gamma_{L} \nu_{e i} / 2}$. Below the 'second threshold' results from the numerical simulation coincide roughly with the results of the linear theory. Above this point the non-linear interaction between the wave and the electrons starts to be dominant and the resulting instability growth rate is lower than the one predicted by the linear theory.

\section{CONCLUSIONS}

Although the simplified version of the numerical simulation should prevent the development of trapped particle instability, the Raman cascading and the forward SRS, the following conclusions can still be made: (1) In accordance with the linear theory we found that a strong instability growth and a lower threshold occur in the thinner plasma of the outer corona. The instability threshold in our particular case lies around $P=10^{15} \mathrm{~W} / \mathrm{cm}^{2}$. (2) The Maxwell-Vlasov model renders two threshold intensities. The lower one is connected with the linear instability threshold, and the temporal evolution in this case is unaffected by the nonlinear electron dynamics. Above the 'second threshold' (in our particular case $P=3 \times 10^{16} \mathrm{~W} / \mathrm{cm}^{2}$ ) the electron dynamics starts to be important and the growth rate is reduced due to the strong nonlinear wave/particle interaction leading to the trapped electron regime (see Fig. 2). (3) Fig. 1(a) demonstrates lower saturation levels for higher laser intensities (caused by stronger waveparticle interaction - compare Fig. 2(b) and (c)), from which we can deduce a lowering of the Raman reflectivity. It is interesting that even with our sparse mode spectrum in the case of highest intensity considered $P=10^{17} \mathrm{~W} / \mathrm{cm}^{2}$ the trapped particle instability still sets on as visualised by the electron phase space shown in Fig. 2(d), see also [6-8].

Support by the Ministry of Education, Youth and Sports of the Czech Republic project No. LC528 and of the grant P205/11/0571 of the Czech Science Foundation is gratefully acknowledged.

\section{References}

[1] T. P., Armstrong, R. C., Harding, G., Knorr, and D., Montgomery, Solution of Vlasov equation by transform methods. In Methods in Computational Physics 9, (B. Alder, S. Fernbach, and M. Rotenberg, Eds., Academic Press, London, 1970), 29-86 


\section{IFSA 2011}

[2] F. C., Grant, and M. R., Feix, Phys. Fluids 10, 696, (1967)

[3] F. C., Grant, and M. R., Feix, Phys. Fluids 10, 1356, (1967)

[4] M., Mašek, and K., Rohlena, Czech. J. Phys. 55 (8), 973, (2005)

[5] M., Mašek, and K., Rohlena, Communications in Nonlinear Science and Numerical Simulation 13, 125, (2008)

[6] M., Mašek, and K., Rohlena, Eur. Phys. J. D 56, 79-90, (2010)

[7] M., Mašek, and K., Rohlena, 35th EPS Conference on Plasma Phys., Hersonissos (9-13 June 2008), http: //epsppd.epfl.ch/Hersonissos/pdf/P5_129.pdf

[8] M., Mašek, and K., Rohlena, Radiation Effects and Defects in Solids 165, 405-411, (2010) 